\title{
Effective formulation reductions for the quadratic assignment problem
}

\author{
Huizhen Zhang* Cesar Beltran-Royo ${ }^{\dagger}$ Miguel Constantino ${ }^{\ddagger}$ \\ $11 / 12 / 2008$
}

\begin{abstract}
In this paper we study two formulation reductions for the quadratic assignment problem (QAP). In particular we apply these reductions to the well known Adams and Johnson [2] integer linear programming formulation of the QAP, which we call formulation IPQAP-I. We analyze two cases: In the first case, we study the effect of constraint reduction. In the second case, we study the effect of variable reduction in the case of a sparse cost matrix. Computational experiments with a set of 32 QAPLIB instances, which range from 12 to 32 locations, are presented. The proposed reductions turned out to be very effective: By applying the new constraint reduction or the new variable reduction to the IPQAP-I formulation, we solved 13 and 23 instances, respectively, compared to the 7 instances solved by formulation IPQAP-I.
\end{abstract}

Key words: quadratic assignment problem, linear integer programming, linear programming relaxation, sparse matrix.

\section{Introduction}

The quadratic assignment problem(QAP) was first proposed by Koopmans and Beckmann in 1957 as a mathematical model related to the location of a set of indivisible economical activities [26]. Consider the problem of assigning $n$ facilities to $n$ locations in such a way that each facility is designated to exactly one location and vice-versa. The objective is to minimize the quadratic interaction cost, a function of the distances and flows between the

\footnotetext{
*zhzzywz@gmail.com, School of Management, University of Shanghai for Science and Technology, Shanghai, China

†Corresponding author: cesar.beltran@urjc.es, Statistics and Operations Research, Rey Juan Carlos University, Madrid, Spain.

${ }_{\ddagger}^{\ddagger}$ miguel.constantino@fc.ul.pt, DEIO/ Centro de Investigação Operacional, Faculdade de Ciências, Universidade de Lisboa, Lisbon, Portugal
} 
facilities, plus the costs associated with allocating a facility to a certain location. Therefore, given three $n \times n$ matrices with real elements $F=\left(f_{i k}\right), D=\left(d_{j l}\right)$ and $C=\left(c_{i j}\right)$, where $f_{i k}$ is the flow between the facility $i$ and facility $k, d_{j l}$ is the distance between the location $j$ and $l$, and $c_{i j}$ is the cost of allocating facility $i$ at location $j$, the QAP can be stated as follows:

$$
\min _{x \in X} \sum_{i, j, k, l=1}^{n} q_{i j k l} x_{i j} x_{k l}+\sum_{i, j=1}^{n} c_{i j} x_{i j}
$$

where $q_{i j k l}=f_{i k} d_{j l}$

$$
x_{i j}= \begin{cases}1 & \text { if facility } i \text { is assigned to location } j \\ 0 & \text { otherwise }\end{cases}
$$

and $X$ is the set of permutation matrices of dimension $n$.

This set of permutations can be defined as:

$$
\begin{aligned}
& X=\left\{x \mid \quad \sum_{j=1}^{n} x_{i j}=1 \quad i \in N\right. \\
& \sum_{i=1}^{n} x_{i j}=1 \quad j \in N \\
& \left.x_{i j} \in\{0,1\} \quad i, j \in N\right\}
\end{aligned}
$$

where $N=\{1, \ldots, n\}$.

Lawler [27] considered a more general QAP, where the $q_{i j k l}$ coefficients in (1.1) are not restricted to flow-distance products, in contrast with the original Koopman-Beckmann formulation.

The QAP has drawn researcher's attention worldwide and extensive research has been done for more than half century. The QAP problem is considered one of the most difficult combinatorial problems: it is $N P$-hard, and even finding an $\varepsilon$-approximate solution is a hard problem [37]. It is surprising the number of fields where the QAP problem can be applied. In addition to its application in facility location, the QAP has been applied in many fields such as printed circuit board assembly process [17], typewriter keyboards and control panels design [34], scheduling [21], numerical analysis [8], and many others. Moreover, many well-known classical combinatorial optimization problems such as the traveling salesman problem, the graph partitioning problem, the maximum clique problem, can also be formulated as special cases of the QAP, see [33] for details.

The advances in theoretical aspects, solution techniques and applications of the QAP have been discussed in more detail, for example, in $[10,5,13,30]$. Regarding recent QAP advances, it is worth it to mention that, during the last years some of the most challenging QAP instances have been solved by combining parallel branch-and-bound algorithms [31, 15] with grid computing [4].

Calculation of lower bounds is an essential component of exact QAP methods, which employ implicit enumeration in a branch and bound framework, see [18, 24, 35]. On the other hand, lower bounds are used to evaluate the quality of solutions produced by heuristic algorithms, like simulated annealing algorithms [14, 32], genetic algorithms(GA) 
$[3,16]$, greedy randomized adaptive search procedure(GRASP) [29], ant colony algorithms [20], and so on. Different QAP bounds have been proposed: Gilmore-Lawler bound, eigenvalue bounds, quadratic programming bounds, LP bounds, polyhedral bounds, semidefinite bounds, among others. More details about QAP lower bounds can be found in $[38,1,28,6,23,39]$.

In this paper we have two main objectives. The first objective is to study the effect of constraint reduction in (linear) integer programming QAP formulations (IPQAP). In the second objective we study the effect of variable and constraint reduction in the case of some null flows (sparse flow matrix).

With the first objective in mind, we present a new LP bound for the QAP, which is more effective than previous LP bounds. It is known that LP and dual-LP bounds are tight for the QAP, but appear to be computationally prohibitive in many cases [5]. To develop the new LP bound, our starting point is the IPQAP formulation of Adams and Johnson [2], that we name IPQAP-I. Then, by virtually dividing by two the number of its constraints, we propose an equivalent formulation, that we name IPQAP-II. This new formulation is less tight than IPQAP-I, but its LP relaxation can be solved much faster. The final result, is that formulation $I P Q A P-I I$ usually requires a $\mathrm{B} \& \mathrm{~B}$ tree with more nodes but more efficient in terms of total solving time.

Regarding the second objective, we have observed that quite a lot of QAP instances have a sparse flow matrix. As far as we know, this fact has not been exploited yet in literature. We study how one can exploit those zero flows. The key point is that in presence of one single zero flow, say $f_{i_{0} k_{0}}$, many coefficient costs $q_{i_{0} j k_{0} l}$ become also zero. We will show how the associated variables $y_{i_{0} j k_{0} l}$ can be eliminated in the QAP formulation. We name $I P Q A P-I I I$ and $I P Q A P-I V$ this reduced variable version of formulations IPQAP-II and $I P Q A P-I$, respectively.

In our numerical experiments, we have used a set of 32 QAPLIB instances [9], that range from 12 to 32 locations, all of them with a sparse flow matrix. The results obtained by the new formulations have been surprisingly remarkable, especially, if we take into account that we have conducted our tests with a standard laptop, CPLEX 9.0 with default parameters and 4 hours of CPU time limit. Within these conditions and by using the IPQAP formulations I, II, III and IV, we have solved up to optimality 7, 13, 21 and 23 QAPLIB instances, respectively.

This paper is organized as follows. In Section 2, we review the Adams and Johnson QAP linearization. In section 3 we study the constraint reduction. In section 4 and 5, we study some variable reductions, especially in the case of some null flows (sparse flow matrix). The numerical experiments are presented in section 6 . Concluding remarks are made in the last section. 


\section{Adams and Johnson linearization}

Adams and Johnson [2] linearization is the well-known linear integer programming QAP formulation:

$$
\begin{aligned}
& \min _{x, y} \sum_{i, j, k, l=1}^{n} q_{i j k l} y_{i j k l}+\sum_{i, j=1}^{n} c_{i j} x_{i j} \\
& \text { s. t. } \quad \sum_{l=1}^{n} y_{i j k l}=x_{i j} \quad i, j, k \in N \\
& \sum_{k=1}^{n} y_{i j k l}=x_{i j} \quad i, j, l \in N \\
& y_{i j k l}=y_{k l i j} \quad i, j, k, l \in N \\
& y_{i j k l} \in\{0,1\} \quad i, j, k, l \in N \\
& x \in X
\end{aligned}
$$

This formulation, that we name IPQAP-I, contains $o\left(n^{4}\right)$ variables and $o\left(n^{4}\right)$ constraints. Although, it produces tight LP bounds, usually it poses an obstacle for efficiently solving QAP instances from medium to large scale. Even to solve the associated LP relaxation can be difficult [36]. Other QAP linearizations can be found in literature: Lawler's linearization [22] as the first one, Kaufmann and Broeckx's linearization [25] has the smallest number of variables and constraints and Frieze and Yadegar's linearization [19], among others.

\section{Formulation reduction by constraint elimina- tion}

In this section we introduce the new formulation IPQAP-II, which corresponds to formulation $I P Q A P-I$ without constraints (2.7), and with half of the constraints (2.6) relaxed into the $\leq$ form, that is:

$$
\begin{aligned}
& \min _{x, y} \sum_{i, j, k, l=1}^{n} q_{i j k l} y_{i j k l}+\sum_{i, j=1}^{n} c_{i j} x_{i j} \\
& \text { s. t. } \quad \sum_{l=1}^{n} y_{i j k l}=x_{i j} \quad i, j, k \in N, i \leq k \\
& \sum_{l=1}^{n} y_{i j k l} \leq x_{i j} \quad i, j, k \in N, i>k \\
& y_{i j k l}=y_{k l i j} \quad i, j, k, l \in N \\
& y_{i j k l} \in\{0,1\} \quad i, j, k, l \in N \\
& x \in X
\end{aligned}
$$

Proposition 3.1 IPQAP-II is a (valid) formulation for the QAP. 
Proof: We consider the following equivalent formulation of the QAP in the $(x, y)$ space (we name it $\left.Q A P^{\prime}\right)$ :

$$
\begin{array}{ll}
\min _{x, y} & \sum_{i, j, k, l=1}^{n} q_{i j k l} y_{i j k l}+\sum_{i, j=1}^{n} c_{i j} x_{i j} \\
\text { s. t. } & x_{i j} x_{k l}=y_{i j k l} \quad \\
& x \in X
\end{array}
$$

Let us name $F_{Q A P^{\prime}}, F_{I}$ and $F_{I I}$ the feasible sets of formulations $Q A P^{\prime}, I P Q A P-I$ and $I P Q A P-I I$, respectively. To prove this proposition, it is enough to prove that $F_{Q A P^{\prime}}=F_{I I}$, since $Q A P^{\prime}$ and $I P Q A P-I I$ have the same objective function.

First, let us see that $F_{Q A P^{\prime}} \subset F_{I I}$. We know that $Q A P^{\prime}$ and IPQAP-I are equivalent formulations and that IPQAP-II is a relaxation of IPQAP-I. Therefore,

$$
F_{Q A P}=F_{I} \subset F_{I I}
$$

Second, let us see that $F_{I I} \subset F_{Q A P^{\prime}}$. We consider $(x, y) \in F_{I I}$ and will prove that $(x, y) \in F_{Q A P^{\prime}}$. Since $x \in X$, it is enough to see that $y_{i j k l}=x_{i j} x_{k l}$ for $1 \leq i, j, k, l \leq n$. Without loss of generality, we assume that $i \leq k$.

We analyze the three possible cases:

1. If $x_{i j} x_{k l}=0$, with $x_{i j}=0$ then $\sum_{l=1}^{n} y_{i j k l}=x_{i j}=0$ by equation (3.12). This implies $y_{i j k l}=0$.

2. If $x_{i j} x_{k l}=0$, with $x_{k l}=0$, we distinguish two subcases:

(a) If $i<k$, then $\sum_{j=1}^{n} y_{k l i j} \leq x_{k l}=0$ by equation (3.13).

(b) If $i=k$, then $\sum_{j=1}^{n} y_{k l i j}=x_{k l}=0$ by equation (3.12).

In both subcases $y_{k l i j}=0$ and therefore $y_{i j k l}=0$.

3. If $x_{i j} x_{k l}=1$ then $x_{i j}=x_{k l}=1$. Let us suppose that $y_{i j k l}=0$ and get a contradiction. If $y_{i j k l}=0$, considering that $\sum_{l=1}^{n} y_{i j k l}=x_{i j}=1$, there must exist $l^{\prime} \in N$ with $l^{\prime} \neq l$ such that $y_{i j k l^{\prime}}=1$. We distinguish two subcases:

(a) If $i<k$, then $1=y_{i j k l^{\prime}}=y_{k l^{\prime} i j} \leq \sum_{j=1}^{n} y_{k l^{\prime} i j} \leq x_{k l^{\prime}} \leq 1$, by equation $(3.13)$.

(b) If $i=k$, then $1=y_{i j k l^{\prime}}=y_{k l^{\prime} i j}=\sum_{j=1}^{n} y_{k l^{\prime} i j}=x_{k l^{\prime}} \leq 1$ by equation (3.12).

In both cases $x_{k l^{\prime}}=1\left(l^{\prime} \neq l\right)$, which contradicts our hypothesis $x_{k l}=1$ together with $\sum_{l=1}^{n} x_{k l}=1$. 
Given an Integer Programming formulation IPQAP, we denote by $L P Q A P$ its Linear Programming (LP) relaxation, i.e., the formulation obtained by replacing the integrality constraints $y_{i j k l} \in\{0,1\}$ for $i, j, k, l \in N$ and $x_{i j} \in\{0,1\}$ for $i, j \in N$ by their continuous counterparts, $0 \leq y_{i j k l} \leq 1$ for $i, j, k, l \in N$ and $0 \leq x_{i j} \leq 1$ for $i, j \in N$ respectively.

In general, the optimal value of the LP relaxation $L P Q A P-I$ is larger than the optimal value of $L P Q A P-I I$, that is, the first formulation is tighter. Nevertheless, $L P Q A P-I I$ has fewer constraints, so it is easier to solve.

\section{Formulation reduction in the case of some null flows}

In this section we assume that the quadratic cost coefficients are proportional to the flow and to the distance, that is, $q_{i j k l}=f_{i k} d_{j l}$. In many QAP instances, there are pairs of facilities $i_{0} k_{0}$ whose flow is zero $\left(f_{i_{0} k_{0}}=0\right)$. In this case, the associated quadratic cost coefficient $q_{i_{0} j k_{0} l}$ vanishes for all locations $j$ and $l$. We call zero flow variables the associated variables $y_{i_{0} j k_{0} l}$. From the objective function point of view it is irrelevant the value of a zero flow variable. One would like to fix them, say, to zero and thus reduce the number of variables. In this context, when we say that we eliminate one variable, we mean that we fix it to zero. However, as we will see in the following proposition, the zero flow variables cannot be eliminated, since they are relevant from the feasible set point of view (in fact any $y_{i j k l}$ is relevant).

Proposition 4.2 In formulations IPQAP-I and IPQAP-II one cannot eliminate any zero flow variable $y_{i j k l}(i \neq k, j \neq l)$.

Proof: (By contradiction.) In formulation IPQAP-I, let us take $x^{0} \in X$ and assume that $x_{i_{0} j_{0}}^{0}=x_{k_{0} l_{0}}^{0}=1\left(i_{0} \neq k_{0}, j_{0} \neq l_{0}\right)$, and $f_{i_{0} k_{0}}=0$. If we eliminate variable $y_{i_{0} j_{0} k_{0} l_{0}}$, by constraint (2.6), we have (without loss of generality, we assume that $\left.i_{0}<k_{0}\right)$ :

$$
\sum_{l=1, l \neq l_{0}}^{n} y_{i_{0} j_{0} k_{0} l}=x_{i_{0} j_{0}}^{0}=1 .
$$

Therefore, there must exist $l_{1} \neq l_{0}$ such that $y_{i_{0} j_{0} k_{0} l_{1}}=1$. Now, by constraints (2.7) and (2.8) we have:

$$
1=y_{i_{0} j_{0} k_{0} l_{1}}=y_{k_{0} l_{1} i_{0} j_{0}} \leq \sum_{i=1}^{n} y_{k_{0} l_{1} i j_{0}}=x_{k_{0} l_{1}}^{0} \leq 1 .
$$

Thus, $x_{k_{0} l_{1}}^{0}=1$ with $l_{1} \neq l_{0}$, which contradicts our hypothesis $x_{k_{0} l_{0}}^{0}=1$ for $x^{0} \in X$.

Similarly, it can be shown that in formulation IPQAP-II one cannot eliminate any zero flow variable $y_{i j k l}(i \neq k, j \neq l)$ either. 
In this proposition, we have seen that, in order to obtain an equivalent QAP formulation, it is not possible to simply eliminate the zero flow variables in formulations IPQAP-I and IPQAP-II. It turns out that, if we eliminate zero flow variables, we also need to eliminate, what we call zero flow constraints (constraints with at least one eliminated zero flow variable). This is done in formulation IPQAP-III, where we reduce formulation $I P Q A P-I I$, by eliminating the zero flow variables and the zero flow constraints. To state IPQAP-III we need to define first the index set of zero flows, i.e.,

$$
F_{0}=\left\{(i, k) \in N \times N \mid f_{i k}=0\right\} .
$$

We assume that the flow matrix is symmetrical and therefore if $(i, k) \in F_{0}$ then $(k, i) \in F_{0}$ as well.

We state the IPQAP-III formulation as follows:

$$
\begin{aligned}
& \min _{x, y} \sum_{i, j, k, l=1, i k \notin F_{0}}^{n} q_{i j k l} y_{i j k l}+\sum_{i, j=1}^{n} c_{i j} x_{i j} \\
& \text { s. t. } \quad \sum_{l=1}^{n} y_{i j k l}=x_{i j} \quad i, j, k \in N, i \leq k, i k \notin F_{0} \\
& \sum_{l=1}^{n} y_{i j k l} \leq x_{i j} \quad i, j, k \in N, i>k, i k \notin F_{0} \\
& y_{i j k l}=y_{k l i j} \quad i, j, k, l \in N, i k \notin F_{0} \\
& y_{i j k l} \in\{0,1\} \quad i, j, k, l \in N, i k \notin F_{0} \\
& x \in X
\end{aligned}
$$

Proposition 4.3 Formulation IPQAP-III is equivalent to IPQAP-II.

Proof: Let $F_{I I}$ and $F_{I I I}$ be the feasible sets of IPQAP-II and IPQAP-III, respectively. Let $V_{I I}$ and $V_{I I I}$ be the sets of feasible objective values of formulations $I P Q A P-I I$ and IPQAP-III, respectively. To prove this proposition, we will show that $V_{I I}=V_{I I I}$, and therefore the minimum of both formulations is the same. Furthermore, we will also show how to compute an optimal IPQAP-II solution once we have an optimal IPQAP-III solution and vice versa.

First, let us show $V_{I I I} \subset V_{I I}$. In fact, we prove something stronger: any solution $(x, y) \in F_{I I I}$ can be extended to a solution $(\widetilde{x}, \widetilde{y}, \bar{y}) \in F_{I I}$ with the same objective value ( $\bar{y}$ correspond to the coordinates $y_{i j k l}$ with $(i, k) \in F_{0}$ and $\left.j, l \in N\right)$.

The procedure is as follows: define $\widetilde{x}=x$ and $\widetilde{y}=y$. To complete the extended solution $(\widetilde{x}, \widetilde{y}, \bar{y})$, the value of the removed variables $\bar{y}_{i j k l}$ for $(i, k) \in F_{0}$ and $i, j \in N$, is given by $\bar{y}_{i j k l}=x_{i j} \times x_{k l}$. Observe that we have $\sum_{l=1}^{n} y_{i j k l}=\sum_{l=1}^{n} x_{i j} x_{k l}=$ $x_{i j} \sum_{l=1}^{n} x_{k l}=x_{i j}$, the last equality follows from (1.2). Hence $(\widetilde{x}, \widetilde{y}, \bar{y})$ satisfies constraints (3.12-3.16), so it is feasible for IPQAP-II. Furthermore, $(\widetilde{x}, \widetilde{y}, \bar{y})$ and $(x, y)$ have the same objective function value since $q_{i j k l}=0$ for all $(i, k) \in F_{0}$ and $j, l \in N$, 
Second, let us show $V_{I I I} \supset V_{I I}$. In fact, we prove something stronger: given any solution $(\widetilde{x}, \widetilde{y}, \bar{y}) \in F_{I I}$ ( $\bar{y}$ correspond to the coordinates $i j k l$ with $(i, k) \in F_{0}$ and $j, l \in N)$, it can be projected to a solution $(x, y) \in F_{I I I}$ with the same objective value. The procedure is as follows: define $x=\widetilde{x}$ and $y=\widetilde{y}$. Obviously, $(x, y)$ is feasible for IPQAP-III and as before, $(x, y)$ and $(\widetilde{x}, \widetilde{y}, \bar{y})$ have the same objective value.

We would obtain a valid statement if in the previous Proposition we replace $I P Q A P-I I$ and IPQAP-III by LPQAP-II and LPQAP-III respectively. In fact, the above proof remains valid in this case, as the integrality of the variables is never used. Hence we may conclude that the LP relaxations LPQAP-II and LPQAP-III are equivalent.

Similarly, we can eliminate the zero flow variables and the zero flow constraints in the formulation IPQAP-I. The reduced IPQAP-I is named as IPQAP-IV:

$$
\begin{aligned}
& \min _{x, y} \sum_{i, j, k, l=1, i k \notin F_{0}}^{n} q_{i j k l} y_{i j k l}+\sum_{i, j=1}^{n} c_{i j} x_{i j} \\
& \text { s. t. } \quad \sum_{l=1}^{n} y_{i j k l}=x_{i j} \quad i, j, k \in N, i k \notin F_{0} \\
& \sum_{k=1}^{n} y_{i j k l}=x_{i j} \quad i, j, l \in N, \quad i \notin I_{0} \\
& y_{i j k l}=y_{k l i j} \quad i, j, k, l \in N, i k \notin F_{0} \\
& y_{i j k l} \in\{0,1\} \quad i, j, k, l \in N, i k \notin F_{0} \\
& x \in X
\end{aligned}
$$

where $I_{0}$ is the the index set: $I_{0}=\left\{i \in N \mid \exists k: f_{i k}=0\right\}$. Furthermore, we can get the following proposition:

Proposition 4.4 IPQAP-IV is a (valid) formulation for the QAP.

Proof: Observe that IPQAP-IV is the same as IPQAP-III with additional constraints (4.25). From Propositions 3.1 and 4.3 it follows that these constraints are satisfied by the feasible set of IPQAP-III. Hence the feasible sets of IPQAP-IV and $I P Q A P-I I I$ are equal. Since the objective function is the same and IPQAP-III is a valid formulation for the QAP, the result follows.

The above proof is not valid if we consider the linear relaxations $L P Q A P-I V$ and LPQAP-III instead of IPQAP-IV and IPQAP-III, because constraints (4.25) may not be redundant to LPQAP-III. Let $\bar{V}_{I}-\bar{V}_{I V}$ denote the optimal values of $I P Q A P-I-I P Q A P-I V$ respectively. We have the following relationship between the LP relaxations:

$$
\bar{V}_{I I I}=\bar{V}_{I I} \leq \bar{V}_{I V} \leq \bar{V}_{I}
$$

and there are instances for which the inequalities are strict (See section 6). 


\section{Further formulation reductions}

In formulations IPQAP, I to IV, we can eliminate many variables, especially we can use only one of the duplicated variables $y_{i j k l}=y_{k l i j}$. We start by reducing the number of variables in formulation IPQAP-II. The symmetry constraints (3.14) imply that we can formulate $I P Q A P-I I$ by using only variables $y_{i j k l}$, with $i \leq k$.

The elimination of the duplicated variables $y_{i j k l}(i>k)$ can be done as follows:

- In the objective function, substitute all variables $y_{i j k l}$ with $i>k$ by $y_{k l i j}$.

- In constraint (3.13) substitute $y_{i j k l}$ with $i>k$ by $y_{k l i j}$

$$
\sum_{l=1}^{n} y_{k l i j} \leq x_{i j} \quad i, j, k \in N, \quad i>k,
$$

and rename the $k l i j$ indexes as $i j k l$

$$
\sum_{j=1}^{n} y_{i j k l} \leq x_{k l} \quad i, k, l \in N, \quad i<k
$$

- Eliminate the symmetry constraints (3.14).

With the above variable reduction, we get an equivalent formulation of IPQAP-II that we call IPQAP-II':

$$
\begin{aligned}
& \min _{x, y} \sum_{1 \leq i \leq k \leq n} \sum_{1 \leq j, l \leq n} q_{i j k l} y_{i j k l}+\sum_{1 \leq k<i \leq n} \sum_{1 \leq j, l \leq n} q_{k l i j} y_{i j k l}+\sum_{i, j=1}^{n} c_{i j} x_{i j} \\
& \text { s. t. } \quad \sum_{l=1}^{n} y_{i j k l}=x_{i j} \quad i, j, k \in N, i \leq k \\
& \sum_{j=1}^{n} y_{i j k l} \leq x_{k l} \quad i, k, l=1 \in N, i<k \\
& y_{i j k l} \in\{0,1\} \quad i, j, k, l \in N, i \leq k \\
& x \in X
\end{aligned}
$$

Further variable reduction can be done in IPQAP-II' for variables $y_{i j k l}$ with $i=k$ or $j=l$ :

1. Case $i=k$ and $j=l$. Considering that for any $(x, y)$ feasible for IPQAP-II', we have $y_{i j i j}=x_{i j}$ for all $i j$ :

(a) In the objective function, substitute all variables $y_{i j i j}$ by $x_{i j}$.

(b) In constraints (5.30) substitute all variables $y_{i j i j}$ by $x_{i j}$.

2. Case $i=k$ and $j \neq l$. Considering that $y_{i j i l}=0$ for all $i$ and $j \neq l$.

(a) In the objective function eliminate the terms ijil for all $i$ and $j \neq l$. 
(b) In constraints (5.30), eliminate the terms $i j i l$ for all $i$ and $j \neq l$. Note that, this term elimination together with substitution 1.(b) implies that in (5.30) we can eliminate all the equations with $i=k$.

3. Case $i \neq k$ and $j=l$. Considering that $y_{i j k j}=0$ for all $j$ and $i \neq k$.

(a) In the objective function eliminate the terms $i j k j$ for all $j$ and $i \neq k$.

(b) In constraints (5.30-5.31), eliminate the terms $i j k j$ for all $j$ and $i \neq k$.

4. Eliminate variables $y_{i j i l}$ and $y_{i j k j}$.

With the above variable reduction, we get an equivalent reduced formulation of $I P Q A P-I I '$, which we call IPQAPR-II:

$$
\begin{aligned}
& \min _{x, y} \sum_{1 \leq i<k \leq n} \sum_{1 \leq j \neq l \leq n} \widetilde{q}_{i j k l} y_{i j k l}+\sum_{i, j=1}^{n} p_{i j} x_{i j} \\
& \text { s. t. } \quad \sum_{l=1, l \neq j}^{n} y_{i j k l}=x_{i j} \quad i, j, k \in N, i<k \\
& \sum_{j=1, j \neq l}^{n} y_{i j k l} \leq x_{k l} \quad i, k, l \in N, i<k \\
& y_{i j k l} \in\{0,1\} \quad i, j, k, l \in N, i<k, l \neq j \\
& x \in X
\end{aligned}
$$

where $\widetilde{q}_{i j k l}=q_{i j k l}+q_{k l i j}$ and $p_{i j}=c_{i j}+q_{i j i j}$.

In the past years, some pioneer QAP researchers performed similar variable reductions in formulation IPQAP-I, to obtain what we call formulation IPQAPR-I (see [5]):

$$
\begin{aligned}
& \min _{x, y} \sum_{1 \leq i<k \leq n} \sum_{1 \leq j \neq l \leq n} \widetilde{q}_{i j k l} y_{i j k l}+\sum_{i, j=1}^{n} p_{i j} x_{i j} \\
& \text { s. t. } \sum_{l=1, l \neq j}^{n} y_{i j k l}=x_{i j} \quad i, j, k \in N, i<k \\
& \sum_{j=1, j \neq l}^{n} y_{i j k l}=x_{k l} \quad i, k, l \in N, i<k \\
& \sum_{k=1}^{i-1} y_{k l i j}+\sum_{k=i+1}^{n} y_{i j k l}=x_{i j} \quad i, j, l \in N, \quad j \neq l \\
& y_{i j k l} \in\{0,1\} \quad i, j, k, l \in N \text { and } k>i \\
& x \in X
\end{aligned}
$$

Finally, by performing the above variable reductions to formulation IPQAP-III and $I P Q A P-I V$, we obtain what we call formulation IPQAPR-III and IPQAPR-IV, 
respectively:

IPQAPR-III :

$$
\begin{aligned}
& \min _{x, y} \sum_{1 \leq i<k \leq n, i k \notin F_{0}} \sum_{1 \leq j \neq l \leq n} \widetilde{q}_{i j k l} y_{i j k l}+\sum_{i, j=1}^{n} p_{i j} x_{i j} \\
& \text { s. t. } \quad \sum_{l=1, l \neq j}^{n} y_{i j k l}=x_{i j} \quad i, j, k \in N, i<k, i k \notin F_{0} \\
& \sum_{j=1, j \neq l}^{n} y_{i j k l} \leq x_{k l} \quad i, k, l \in N, i<k, i k \notin F_{0} \\
& y_{i j k l} \in\{0,1\} \quad i, j, k, l \in N, i<k, l \neq j, i k \notin F_{0} \\
& x \in X
\end{aligned}
$$

$I P Q A P R-I V$ :

$$
\begin{aligned}
& \min _{x, y} \sum_{1 \leq i<k \leq n, i k \notin F_{0}} \sum_{1 \leq j \neq l \leq n} \widetilde{q}_{i j k l} y_{i j k l}+\sum_{i, j=1}^{n} p_{i j} x_{i j} \\
& \text { s. t. } \quad \sum_{l=1, l \neq j}^{n} y_{i j k l}=x_{i j} \quad i, j, k \in N, i<k, i k \notin F_{0} \\
& \sum_{j=1, j \neq l}^{n} y_{i j k l}=x_{k l} \quad i, k, l \in N, i<k, i k \notin F_{0} \\
& \sum_{k=1}^{i-1} y_{k l i j}+\sum_{k=i+1}^{n} y_{i j k l}=x_{i j} \quad i, j, l \in N, \quad j \neq l, \quad i \notin I_{0} \\
& y_{i j k l} \in\{0,1\} \quad i, j, k, l \in N \quad k>i, \quad j \neq l, \quad i k \notin F_{0} \\
& x \in X
\end{aligned}
$$

\section{Numerical experiments}

In this section, we present the experimental results obtained with the formulations IPQAPR-I, IPQAPR-II, IPQAPR-III and IPQAPR-IV, applied to some sparse flow-input matrices instances from QAPLIB [9]. The experiments were conducted on a laptop with an Intel Pentium M-1.70GHz processor and with 512 MB RAM. Matlab 7.0 was used to set up the formulations and Cplex 9.0 was used to solve the resulting IP instances. Cplex default parameters were used throughout. The program was allowed to run for 4 hours at most. The set up time (Matlab) and the IP solving time (Cplex) are reported.

In table 1 we summarize some characteristics of the instances used in the tests, namely the density of the flow-input matrices (DFM), which is defined as the percent of the number of non-zero elements in the matrix. We also report the dimension of 
each formulation IPQAPR-I, IPQAPR-II, IPQAPR-III and IPQAPR-IV, namely the number of variables $x_{i j}\left(\mathrm{Nb}\right.$ of $x$ ) and $y_{i j k l}(\mathrm{Nb}$ of $y$ ), and the number of constraints ( $\mathrm{Nb}$ of constraints).

In table 2 we present the value of the objective function of the best integer solution (Cost) obtained for each problem instance, as well as the gap measured by Cplex directly. The gap is given by the formula Gap $=\frac{\text { Cost-bestnode }}{\text { Cost }} \times 100 \%$, where bestnode denotes the lower bound obtained by Cplex.

In table 3 we present the linear programming relaxation values (LPcost), gaps, $L P g a p=\frac{o p t v-L P c o s t}{o p t v} \times 100 \%$, where optv is the optimal objective value, and the CPU times spent in solving the linear programming relaxation of every tested instances.

The corresponding CPU times for setting up the formulations and solving the mixed integer linear programs are shown in table 4 . In this table we also present the CPU time given in the literature for some of the tested instances, with special purpose exact algorithms. Unfortunately, we didn't find the CPU time for solving the instances Esc32a - Esc32d, Esc32g and Esc32h with exact algorithms, so they are absent in the table 4. For the instances, Chr12a-Chr25a, Esc16a-Esc16j, Esc32e and Esc32f, the literature CPU times are obtained in [11], [12] and [7], respectively.

We make the following observation remarks regarding the computational results presented in tables 1- 4 :

- As expected, formulation $I P Q A P R$ - II has considerably fewer constraints than formulation IPQAPR-I or IPQAP-I. The reduced formulations IPQAPR-IV and $I P Q A P R$ - $I I I$ have the same variables, and in most cases they have the same number of constraints, except for instance Esc16h, as shown in table 1. In all but this instance, $I_{0}=N$, that is, for all $i \in N$ there exists $k$ such that the flow $f_{i k}$ is null.

- The linear relaxation bounds obtained with formulation $I P Q A P R$-I are in general better than the ones obtained with formulations IPQAPR-II, as expected. Nevertheless, the integer programming solutions obtained with $I P Q A P R$ $I I$, shown in table 2 , are better or at least with equal value than the solutions obtained by IPQAPR-I. On the other hand the number of nodes in the Branch and Bound tree is larger for IPQAPR-II. Although the LP relaxation is weaker, it can be solved faster, so more nodes can be searched, leading to a superior performance of IPQAPR-II over IPQAPR-I. For the 32 tested instances, 7 and 20 optimal solutions are computed with formulations IPQAPR-I and $I P Q A P R-I I$ respectively, and for 7 and 13 of them optimality has been proved.

- When we look at the results obtained with IPQAPR-III and IPQAPR-IV we see that these formulations outperform IPQAPR-I and IPQAPR-II. Here, a 
smaller number of variables makes the LP relaxations much easier to solve, allowing an even larger Branch and Bound tree within the time limits. For those instances solved to optimality, the LP CPU times as shown in table 3 and the IP CPU times shown in table 4 are smaller for formulations IPQAPR$I I I$ and IPQAPR-IV. For the 32 tested instances, 26 and 27 optimal solutions are computed with formulations IPQAPR-III and IPQAPR-IV respectively, and for 21 and 23 of them optimality has been proved.

- The results obtained with IPQAPR-III and IPQAPR-IV are very similar. As it was mentioned before, $I_{0}=N$ for all instances except for Esc16h. This means these two formulations only differ on constraints (5.47) and (5.52). Observe that for all instances except for Esc16h the LP bounds are the same.

From these observations, we can assert that the formulation IPQAPR-I has a tighter LP bound than the formulations IPQAPR-II, IPQAPR-III and IPQAPR$I V$. Although the LP relaxation of the formulation IPQAPR-II, IPQAPR-III and $I P Q A P R-I V$ may be not so tight, the reduced number of variables and constraints make them much easier to solve. Even though more Branch and Bound nodes are needed, the instances are solved in a shorter time, or better solutions are obtained within the time limit.

Regardless that IPQAPR-II and IPQAPR-III are LP-equivalent, and the linear programming relaxation values of $I P Q A P R-I V$ are worse than $I P Q A P R$ - $I$, it is clear that a lot of time and resources are saved by eliminating the zero flow variables and constraints to reduce the dimensions of the problems in the formulation IPQAPR$I I I$ and IPQAPR-IV. For example, there are absolutely zero flows between 12 of the facilities in the flow matrix of the instance Esc32c. Thus, for any placement of the remaining 20 facilities in the 32 locations, there are $12 !=476,001,600$ solutions with exactly the same value. This multiplicity of solutions is avoided in IPQAPR-III and $I P Q A P R-I V$.

\section{Concluding remarks}

In this paper we presented Integer Programming Formulations obtained by eliminating some variables and/or constraints from the QAP formulation of Adams and Johnson [2]. Although the reduced formulations are less tight, they have considerably fewer variables and constraints, so the LP relaxations are much easier to solve. As a result, using IPQAPR-II, IPQAPR-III and IPQAPR-IV proved to be overall more effective in solving a set of instances from the literature.

The effectiveness of some of the QAP linearizations depends strongly on the sparsity of the cost matrix. When the cost coefficients are given by the product of a 
Table 1: Dimensions of the QAP tested instances implemented with the formulations IPQAPR-I, IPQAPR-II, IPQAPR-III and IPQAPR-IV

\begin{tabular}{|c|c|c|c|c|c|c|c|c|c|}
\hline \multirow{2}{*}{ Ins. } & \multirow{2}{*}{$\mathrm{n}$} & \multirow{2}{*}{$\begin{array}{c}\text { DFM } \\
(\%)\end{array}$} & \multirow{2}{*}{$\begin{array}{c}\text { No. of } \\
x\end{array}$} & \multicolumn{2}{|c|}{ No. of $y$} & \multicolumn{4}{|c|}{ No. of constraints } \\
\hline & & & & $\mathrm{R}-\mathrm{I}^{a} / \mathrm{R}-\mathrm{II}$ & R-III/R-IV & R-I & R-IV & R-II & R-III \\
\hline Chr12a & 12 & 15.28 & 144 & 8712 & 1452 & 3192 & 288 & 1608 & 288 \\
\hline Chr12b & 12 & 15.28 & 144 & 8712 & 1452 & 3192 & 288 & 1608 & 288 \\
\hline Chr12c & 12 & 15.28 & 144 & 8712 & 1452 & 3192 & 288 & 1608 & 288 \\
\hline Chr15a & 15 & 12.44 & 225 & 22050 & 2940 & 6330 & 450 & 3180 & 450 \\
\hline Chr15b & 15 & 12.44 & 225 & 22050 & 2940 & 6330 & 450 & 3180 & 450 \\
\hline Chr15c & 15 & 12.44 & 225 & 22050 & 2940 & 6330 & 450 & 3180 & 450 \\
\hline Chr18a & 18 & 10.49 & 324 & 46818 & 5202 & 11052 & 648 & 5544 & 648 \\
\hline Chr18b & 18 & 10.49 & 324 & 46818 & 5202 & 11052 & 648 & 5544 & 648 \\
\hline Chr20a & 20 & 9.50 & 400 & 72200 & 7220 & 15240 & 800 & 7640 & 800 \\
\hline Chr20b & 20 & 9.50 & 400 & 72200 & 7220 & 15240 & 800 & 7640 & 800 \\
\hline Chr20c & 20 & 9.50 & 400 & 72200 & 7220 & 15240 & 800 & 7640 & 800 \\
\hline Chr22a & 22 & 8.68 & 484 & 106722 & 9702 & 20372 & 968 & 10208 & 968 \\
\hline Chr22b & 22 & 8.68 & 484 & 106722 & 9702 & 20372 & 968 & 10208 & 968 \\
\hline Chr25a & 25 & 7.68 & 625 & 180000 & 14400 & 30050 & 1250 & 15050 & 1250 \\
\hline P.ave. $^{b}$ & 17.6 & 11.3 & 324.9 & 56854.7 & 5646.0 & 11941.7 & 649.7 & 5988.4 & 649.7 \\
\hline Esc16a & 16 & 29.69 & 256 & 28800 & 9120 & 7712 & 1248 & 3872 & 1248 \\
\hline Esc16b & 16 & 71.88 & 256 & 28800 & 22080 & 7712 & 2976 & 3872 & 2976 \\
\hline Esc16c & 16 & 39.84 & 256 & 28800 & 12240 & 7712 & 1664 & 3872 & 1664 \\
\hline Esc16d & 16 & 16.41 & 256 & 28800 & 5040 & 7712 & 704 & 3872 & 704 \\
\hline Esc16e & 16 & 16.41 & 256 & 28800 & 5040 & 7712 & 704 & 3872 & 704 \\
\hline Esc16f & 16 & 0.00 & 256 & 28800 & 0 & 7712 & 32 & 3872 & 32 \\
\hline Esc16g & 16 & 16.41 & 256 & 28800 & 5040 & 7712 & 704 & 3872 & 704 \\
\hline Esc16h & 16 & 89.84 & 256 & 28800 & 27600 & 7712 & 6112 & 3872 & 3712 \\
\hline Esc16i & 16 & 11.72 & 256 & 28800 & 3600 & 7712 & 512 & 3872 & 512 \\
\hline Esc16j & 16 & 9.38 & 256 & 28800 & 2880 & 7712 & 416 & 3872 & 416 \\
\hline P.ave. & 16 & 30.2 & 256.0 & 28800.0 & 9264.0 & 7712.0 & 1507.2 & 3872.0 & 1267.2 \\
\hline Esc32a & 32 & 14.45 & 1024 & 492032 & 73408 & 63552 & 4800 & 31808 & 4800 \\
\hline Esc32b & 32 & 21.09 & 1024 & 492032 & 107136 & 63552 & 6976 & 31808 & 6976 \\
\hline Esc32c & 32 & 25.59 & 1024 & 492032 & 129952 & 63552 & 8448 & 31808 & 8448 \\
\hline Esc32d & 32 & 17.58 & 1024 & 492032 & 89280 & 63552 & 5824 & 31808 & 5824 \\
\hline Esc32e & 32 & 1.17 & 1024 & 492032 & 5952 & 63552 & 448 & 31808 & 448 \\
\hline Esc32f & 32 & 1.17 & 1024 & 492032 & 5952 & 63552 & 448 & 31808 & 448 \\
\hline Esc32g & 32 & 1.76 & 1024 & 492032 & 8928 & 63552 & 640 & 31808 & 640 \\
\hline Esc32h & 32 & 27.54 & 1024 & 492032 & 139872 & 63552 & 9088 & 31808 & 9088 \\
\hline P.ave. & 32.0 & 13.8 & 1024.0 & 492032.0 & 70060.0 & 63552.0 & 4584.0 & 31808.0 & 4584.0 \\
\hline T.ave. ${ }^{c}$ & 20.7 & 17.8 & 478.0 & 156882.0 & 22880.0 & 23523.0 & 1901.0 & 11782.0 & 1826.0 \\
\hline
\end{tabular}

${ }^{a}$ In these tables, R-I, R-II, R-III and R-IV signify IPQAPR-I, IPQAPR-II, IPQAPR-III and IPQAPRIV, respectively.

${ }^{b}$ In this paper, P. ave. is the abbreviation for partial average.

${ }^{c} \mathrm{~T}$. ave. is the abbreviation for total average. 
Table 2: (Mixed) Integer programming results for the tested QAP instances implemented with the formulations IPQAPR-I, IPQAPR-II, IPQAPR-III and IPQAPR-IV (-: no integer feasible solution found in the limited running time)

\begin{tabular}{|c|c|c|c|c|c|c|c|c|c|c|c|c|c|}
\hline \multirow{3}{*}{ Ins. } & \multirow{3}{*}{$\begin{array}{c}\text { Opt. or } \\
\text { B.known } \\
\text { Sol. }\end{array}$} & \multicolumn{8}{|c|}{ (Mixed) IP } & & & & \\
\hline & & \multicolumn{2}{|c|}{ R-I } & \multicolumn{2}{|c|}{ R-IV } & \multicolumn{2}{|c|}{ R-II } & \multicolumn{2}{|c|}{ R-III } & \multicolumn{4}{|c|}{ No. of nodes } \\
\hline & & Cost & $\begin{array}{c}\text { B\&B } \\
\operatorname{Gap}(\%)\end{array}$ & Cost & $\begin{array}{c}\text { B\&B } \\
\text { Gap(\%) }\end{array}$ & Cost & $\begin{array}{c}\text { B\&B } \\
\text { Gap(\%) }\end{array}$ & Cost & $\begin{array}{c}\text { B\&B } \\
\text { Gap(\%) }\end{array}$ & R-I & R-IV & R-II & R-III \\
\hline Chr12a & 9552 & 9552 & 0.0 & 9552 & 0.0 & 9552 & 0.0 & 9552 & 0.0 & 1 & 9 & 741 & 36 \\
\hline Chr12b & 9742 & 9742 & 0.0 & 9742 & 0.0 & 9742 & 0.0 & 9742 & 0.0 & 1 & 120 & 1295 & 21 \\
\hline Chr12c & 11156 & 11156 & 0.0 & 11156 & 0.0 & 11156 & 0.0 & 11156 & 0.0 & 1 & 60 & 889 & 100 \\
\hline Chr15a & 9896 & 9896 & 0.0 & 9896 & 0.0 & 9896 & 0.0 & 9896 & 0.0 & 15 & 60 & 3997 & 127 \\
\hline Chr15b & 7990 & 7990 & 0.0 & 7990 & 0.0 & 7990 & 0.0 & 7990 & 0.0 & 1 & 138 & 2341 & 588 \\
\hline Chr15c & 9504 & 9504 & 0.0 & 9504 & 0.0 & 9504 & 0.0 & 9504 & 0.0 & 1 & 1 & 1 & 1 \\
\hline Chr18a & 11098 & - & - & 11098 & 0.0 & 11098 & 0.0 & 11098 & 0.0 & - & 58 & 941 & 71 \\
\hline Chr18b & 1534 & - & - & 1534 & 0.0 & 1534 & 0.0 & 1534 & 0.0 & - & 4 & 13 & 8 \\
\hline Chr20a & 2192 & - & - & 2192 & 0.0 & 2192 & 0.0 & 2192 & 0.0 & - & 414 & 1168 & 413 \\
\hline Chr20b & 2298 & - & - & 2298 & 0.0 & 2298 & 0.0 & 2298 & 0.0 & - & 655 & 598 & 1431 \\
\hline Chr20c & 14142 & - & - & 14142 & 0.0 & 15100 & 39.1 & 14142 & 0.0 & - & 2110 & 4401 & 17708 \\
\hline Chr22a & 6156 & - & - & 6156 & 0.0 & 6156 & 0.0 & 6156 & 0.0 & - & 505 & 1374 & 230 \\
\hline Chr22b & 6194 & - & - & 6194 & 0.0 & 6194 & 0.0 & 6194 & 0.0 & - & 72 & 605 & 230 \\
\hline Chr25a & 3796 & - & - & 3796 & 0.0 & 5432 & 39.7 & 3796 & 0.0 & - & 1872 & 441 & 5164 \\
\hline P.ave. & 7517.86 & - & - & 7518 & 0.0 & 7703.14 & 5.6 & 7518 & 0.0 & - & 434 & 1343 & 1866 \\
\hline Esc16a & 68 & 100 & 52.0 & 68 & 14.2 & 68 & 100.0 & 68 & 35.5 & 1 & 156136 & 5822 & 155689 \\
\hline Esc16b & 292 & 314 & 11.5 & 292 & 97.3 & 294 & 100.0 & 292 & 100.0 & 1 & 9276 & 936 & 1590 \\
\hline Esc16c & 160 & - & - & 160 & 77.5 & 162 & 95.1 & 162 & 77.5 & - & 67056 & 8120 & 55170 \\
\hline Esc16d & 16 & 38 & 89.5 & 16 & 0.0 & 16 & 100.0 & 16 & 0.0 & 1 & 260927 & 1071 & 92691 \\
\hline Esc16e & 28 & 52 & 73.1 & 28 & 0.0 & 28 & 100.0 & 28 & 0.0 & 1 & 42637 & 3748 & 51465 \\
\hline Esc16f & 0 & 0 & 0.0 & 0 & 0.0 & 0 & 0.0 & 0 & 0.0 & 1 & 1 & 1 & 1 \\
\hline Esc16g & 26 & 46 & 69.6 & 26 & 0.0 & 26 & 76.9 & 26 & 0.0 & 1 & 10001 & 2847 & 9601 \\
\hline Esc16h & 996 & - & - & 996 & 30.7 & 996 & 99.8 & 996 & 99.7 & - & 1 & 10625 & 11881 \\
\hline Esc16i & 14 & 54 & 100.0 & 14 & 0.0 & 14 & 100.0 & 14 & 0.0 & 1 & 4072 & 3631 & 5211 \\
\hline Esc16j & 8 & 26 & 92.3 & 8 & 0.0 & 8 & 62.5 & 8 & 0.0 & 1 & 691 & 2935 & 418 \\
\hline P.ave. & 160.80 & - & - & 161 & 22.0 & 161.20 & 83.4 & 161.00 & 31.3 & - & 55080 & 3974 & 38372 \\
\hline Esc32a & $130\left(\mathrm{~B}^{a}\right)$ & - & - & 194 & 100.0 & - & - & 244 & 100.0 & - & 91 & - & 140 \\
\hline Esc32b & 168(B) & - & - & 300 & 100.0 & - & - & 400 & 100.0 & - & 21 & . & 17 \\
\hline Esc32c & $642(\mathrm{~B})$ & - & - & 736 & 100.0 & - & - & 716 & 100.0 & - & 26 & - & 31 \\
\hline Esc32d & $200(\mathrm{~B})$ & - & - & 236 & 100.0 & - & - & 256 & 100.0 & - & 71 & - & 136 \\
\hline Esc32e & 2 & - & - & 2 & 0.0 & - & - & 2 & 100.0 & - & 367 & - & 3643725 \\
\hline Esc32f & 2 & - & - & 2 & 0.0 & - & - & 2 & 100.0 & - & 367 & - & 3643577 \\
\hline Esc32g & 6 & - & - & 6 & 0.0 & - & - & 6 & 0.0 & - & 7742 & - & 9064 \\
\hline Esc32h & $438(\mathrm{~B})$ & - & - & 532 & 100.0 & - & - & 534 & 100.0 & - & 15 & - & 34 \\
\hline P.ave. & 198.50 & - & - & 251 & 62.5 & - & - & 270 & 87.5 & - & 1088 & - & 912091 \\
\hline T.ave. & 3388.94 & - & - & 3402 & 22.5 & - & - & 3407 & 31.7 & - & 17674 & - & 240830 \\
\hline
\end{tabular}

${ }^{a} \mathrm{~B}$ means the value is the best known solution. 
Table 3: Linear programming relaxation results for the tested QAP instances implemented with the formulations IPQAPR-I, IPQAPR-II, IPQAPR-III and IPQAPR-IV

\begin{tabular}{|c|c|c|c|c|c|c|c|c|c|c|c|c|}
\hline \multirow{3}{*}{ Ins. } & \multicolumn{8}{|c|}{ LP relaxation } & & & & \\
\hline & \multicolumn{2}{|c|}{ R-I } & \multicolumn{2}{|c|}{ R-IV } & \multicolumn{2}{|c|}{ R-II } & \multicolumn{2}{|c|}{ R-III } & \multicolumn{4}{|c|}{ LP CPU Time (Sec.) } \\
\hline & LPcost & $\begin{array}{c}\text { LPgap } \\
(\%)\end{array}$ & LPcost & $\begin{array}{c}\text { LPgap } \\
(\%)\end{array}$ & LPcost & $\begin{array}{c}\text { LPgap } \\
(\%)\end{array}$ & LPcost & $\begin{array}{c}\text { LPgap } \\
(\%)\end{array}$ & R-I & R-IV & R-II & R-III \\
\hline Chr12a & 9552.0 & 0.0 & 8593.1 & 10.0 & 8593.1 & 10.0 & 8593.1 & 10.0 & 19.8 & 0.0 & 0.8 & 0.0 \\
\hline Chr12b & 9742.0 & 0.0 & 7184.0 & 26.3 & 7184.0 & 26.3 & 7184.0 & 26.3 & 15.9 & 0.0 & 0.9 & 0.0 \\
\hline Chr12c & 11156.0 & 0.0 & 10042.7 & 10.0 & 10042.7 & 10.0 & 10042.7 & 10.0 & 33.4 & 0.0 & 0.8 & 0.0 \\
\hline Chr15a & 9513.1 & 3.9 & 8621.9 & 12.9 & 8621.9 & 12.9 & 8621.9 & 12.9 & 1319.6 & 0.1 & 5.0 & 0.1 \\
\hline Chr15b & 7990.0 & 0.0 & 5141.0 & 35.7 & 5141.0 & 35.7 & 5141.0 & 35.7 & 558.6 & 0.1 & 6.0 & 0.1 \\
\hline Chr15c & 9504.0 & 0.0 & 9504.0 & 0.0 & 9504.0 & 0.0 & 9504.0 & 0.0 & 145.4 & 0.1 & 3.5 & 0.1 \\
\hline Chr18a & 10758.3 & 3.1 & 9515.3 & 14.3 & 9515.3 & 14.3 & 9515.3 & 14.3 & 11206.6 & 0.2 & 13.5 & 0.2 \\
\hline Chr18b & - & - & 1534.0 & 0.0 & 1534.0 & 0.0 & 1534.0 & 0.0 & $14400(*)^{a}$ & 0.1 & 55.4 & 0.2 \\
\hline Chr20a & - & - & 2156.0 & 1.6 & 2156.0 & 1.6 & 2156.0 & 1.6 & $14400\left(^{*}\right)$ & 0.2 & 181.7 & 0.3 \\
\hline Chr20b & - & - & 2242.9 & 2.4 & 2242.9 & 2.4 & 2242.9 & 2.4 & $14400(*)$ & 0.5 & 342.2 & 0.5 \\
\hline Chr20c & - & - & 8816.6 & 37.7 & 8816.6 & 37.7 & 8816.6 & 37.7 & $14400\left(^{*}\right)$ & 0.3 & 130.7 & 0.4 \\
\hline Chr22a & - & - & 5993.6 & 2.6 & 5993.6 & 2.6 & 5993.6 & 2.6 & $14400\left(^{*}\right)$ & 0.5 & 189.4 & 0.6 \\
\hline Chr22b & - & - & 6099.0 & 1.5 & 6099.0 & 1.5 & 6099.0 & 1.5 & $14400(*)$ & 0.6 & 82.1 & 0.6 \\
\hline Chr25a & - & - & 3272.0 & 13.8 & 3272.0 & 13.8 & 3272.0 & 13.8 & $14400\left(^{*}\right)$ & 0.9 & 707.4 & 1.1 \\
\hline P.ave. & - & - & 6336.9 & 12.1 & 6336.9 & 12.1 & 6336.9 & 12.1 & 8150.0 & 0.3 & 122.8 & 0.3 \\
\hline Esc16a & 48.0 & 29.4 & 0.0 & 100.0 & 0.0 & 100.0 & 0.0 & 100.0 & 6709.9 & 1.9 & 15.9 & 1.1 \\
\hline Esc16b & 278.0 & 4.8 & 0.0 & 100.0 & 0.0 & 100.0 & 0.0 & 100.0 & 8869.6 & 12.1 & 14.4 & 6.3 \\
\hline Esc16c & - & - & 0.0 & 100.0 & 0.0 & 100.0 & 0.0 & 100.0 & $14400(*)$ & 2.9 & 13.8 & 1.7 \\
\hline Esc16d & 4.0 & 75.0 & 0.0 & 100.0 & 0.0 & 100.0 & 0.0 & 100.0 & 9614.6 & 0.3 & 13.8 & 0.2 \\
\hline Esc16e & 14.0 & 50.0 & 0.0 & 100.0 & 0.0 & 100.0 & 0.0 & 100.0 & 6550.5 & 0.2 & 15.2 & 0.2 \\
\hline Esc16f & 0.0 & 0.0 & 0.0 & 0.0 & 0.0 & 0.0 & 0.0 & 0.0 & 4969.2 & 0.0 & 14.4 & 0.0 \\
\hline Esc16g & 14.0 & 46.2 & 0.0 & 100.0 & 0.0 & 100.0 & 0.0 & 100.0 & 5449.4 & 0.3 & 16.4 & 0.2 \\
\hline Esc16h & 704.0 & 29.3 & 690.0 & 30.7 & 0.0 & 100.0 & 0.0 & 100.0 & 7846.0 & 1532.8 & 14.1 & 12.9 \\
\hline Esc16i & 0.0 & 100.0 & 0.0 & 100.0 & 0.0 & 100.0 & 0.0 & 100.0 & 3547.3 & 0.1 & 14.6 & 0.1 \\
\hline Esc16j & 2.0 & 75.0 & 0.0 & 100.0 & 0.0 & 100.0 & 0.0 & 100.0 & 5023.1 & 0.1 & 13.8 & 0.1 \\
\hline P.ave. & - & - & 69.0 & 83.1 & 0.0 & 90.0 & 0.0 & 90.0 & 7298.0 & 155.1 & 14.6 & 2.3 \\
\hline Esc32a & - & - & 0.0 & 100.0 & - & - & 0.0 & 100.0 & $14400(*)$ & 217.3 & $14400(*)$ & 94.5 \\
\hline Esc $32 b$ & - & - & 0.0 & 100.0 & - & - & 0.0 & 100.0 & $14400(*)$ & 756.3 & $14400(*)$ & 285.8 \\
\hline Esc32c & - & - & 0.0 & 100.0 & - & - & 0.0 & 100.0 & $14400(*)$ & 845.6 & $14400(*)$ & 402.8 \\
\hline Esc32d & - & - & 0.0 & 100.0 & - & - & 0.0 & 100.0 & $14400\left(^{*}\right)$ & 345.0 & $14400\left(^{*}\right)$ & 151.8 \\
\hline Esc32e & - & - & 0.0 & 100.0 & - & - & 0.0 & 100.0 & $14400(*)$ & 0.1 & $14400(*)$ & 0.1 \\
\hline Esc32f & - & - & 0.0 & 100.0 & - & - & 0.0 & 100.0 & $14400(*)$ & 0.1 & $14400(*)$ & 0.1 \\
\hline Esc32g & - & - & 0.0 & 100.0 & - & - & 0.0 & 100.0 & $14400(*)$ & 0.2 & $14400\left(^{*}\right)$ & 0.1 \\
\hline Esc32h & - & - & 0.0 & 100.0 & - & - & 0.0 & 100.0 & $14400(*)$ & 1159.5 & $14400\left(^{*}\right)$ & 471.2 \\
\hline P.ave. & - & - & 0.0 & 100.0 & - & - & 0.0 & 100.0 & $14400(*)$ & 415.5 & $14400(*)$ & 175.8 \\
\hline T.ave. & - & - & 2793.9 & 73.8 & - & - & 2772.4 & 58.4 & 9446.2 & 152.4 & 3658.3 & 44.8 \\
\hline
\end{tabular}

${ }^{a}$ The CPU time limit, 14400 seconds, was reached. 
Table 4: The CPU times spent of solving the tested QAP instances implemented with the formulations IPQAPR-I, IPQAPR-II, IPQAPR-III and IPQAPR-IV

\begin{tabular}{|c|c|c|c|c|c|c|c|c|c|}
\hline \multirow{2}{*}{ Ins. } & \multicolumn{4}{|c|}{ Formulation setup time (Sec.) } & \multicolumn{4}{|c|}{ IP CPU Time (Sec.) } & \multirow{2}{*}{ Litera. $^{a}$} \\
\hline & R-I & R-IV & R-II & R-III & R-I & R-IV & R-II & R-III & \\
\hline Chr12a & 5.6 & 0.8 & 1.8 & 0.5 & 23.7 & 0.6 & 49.4 & 1.0 & 9.4 \\
\hline Chr12b & 5.5 & 0.7 & 1.9 & 0.3 & 36.8 & 0.9 & 126.5 & 1.0 & 2.8 \\
\hline Chr12c & 5.3 & 0.7 & 1.7 & 0.3 & 53.7 & 0.8 & 67.8 & 1.2 & 1.2 \\
\hline Chr15a & 23.4 & 1.7 & 7.1 & 0.8 & $14400(*)^{b}$ & 2.1 & 720.4 & 3.2 & 61.3 \\
\hline Chr15b & 23.5 & 1.7 & 7.2 & 0.9 & 741.1 & 2.9 & 793.7 & 7.9 & 28.0 \\
\hline Chr15c & 23.4 & 1.5 & 7.0 & 0.8 & 190.9 & 0.1 & 3.7 & 0.1 & 11.7 \\
\hline Chr18a & 109.7 & 3.7 & 25.3 & 2.2 & $14400\left(^{*}\right)$ & 4.8 & 974.8 & 6.6 & 89.7 \\
\hline Chr18b & 98.8 & 3.4 & 26.2 & 2.4 & $14400(*)$ & 2.5 & 835.6 & 3.6 & 37.1 \\
\hline Chr20a & 320.5 & 6.2 & 57.0 & 4.3 & $14400(*)$ & 25.8 & 8691.8 & 28.7 & 181.4 \\
\hline Chr20b & 322.6 & 5.7 & 57.1 & 3.8 & $14400\left(^{*}\right)$ & 33.0 & 7237.1 & 56.0 & 56.2 \\
\hline Chr20c & 318.8 & 6.4 & 57.2 & 4.5 & $14400\left(^{*}\right)$ & 69.1 & $14400\left(^{*}\right)$ & 313.6 & 138.0 \\
\hline Chr22a & 920.6 & 10.8 & 136.9 & 8.1 & $14400\left(^{*}\right)$ & 30.1 & 10346.7 & 21.4 & 166.3 \\
\hline $\mathrm{Chr} 22 \mathrm{~b}$ & 922.8 & 10.2 & 137.8 & 7.4 & $14400\left(^{*}\right)$ & 9.7 & 4186.7 & 15.1 & 143.3 \\
\hline Chr25a & 3319.0 & 26.0 & 585.2 & 17.9 & $14400\left(^{*}\right)$ & 121.1 & $14400\left(^{*}\right)$ & 274.0 & 333.3 \\
\hline P.ave. & 458.5 & 5.7 & 79.3 & 3.9 & 9331.9 & 21.7 & 4488.1 & 52.4 & 90.0 \\
\hline Esc16a & 37.3 & 5.1 & 11.5 & 3.2 & $14400\left(^{*}\right)$ & $14400\left(^{*}\right)$ & $14400\left(^{*}\right)$ & $14400\left(^{*}\right)$ & 65.0 \\
\hline Esc16b & 37.3 & 12.9 & 11.3 & 7.7 & $14400\left(^{*}\right)$ & $14400(*)$ & $14400(*)$ & $14400(*)$ & 546.0 \\
\hline Esc16c & 37.4 & 6.6 & 10.9 & 4.0 & $14400\left(^{*}\right)$ & $14400(*)$ & $14400(*)$ & $14400(*)$ & 3990.0 \\
\hline Esc16d & 37.3 & 2.8 & 10.9 & 1.6 & $14400(*)$ & 2685.0 & $14400\left(^{*}\right)$ & 1334.5 & 492.0 \\
\hline Esc16e & 38.2 & 2.9 & 10.9 & 1.7 & $14400\left(^{*}\right)$ & 1001.7 & $14400\left(^{*}\right)$ & 1086.7 & 66.0 \\
\hline Esc16f & 37.3 & 0.5 & 11.6 & 0.2 & 4811.4 & 0.0 & 14.7 & 0.0 & 0.0 \\
\hline Esc16g & 37.4 & 2.9 & 11.6 & 1.8 & $14400\left(^{*}\right)$ & 348.2 & $14400\left(^{*}\right)$ & 301.5 & 7.0 \\
\hline Esc16h & 37.4 & 30.2 & 11.6 & 11.0 & $14400(*)$ & $14400\left(^{*}\right)$ & $14400\left(^{*}\right)$ & $14400\left(^{*}\right)$ & 22082.0 \\
\hline Esc16i & 37.3 & 2.1 & 11.6 & 1.2 & $14400(*)$ & 65.2 & $14400\left(^{*}\right)$ & 65.2 & 14.0 \\
\hline Esc16j & 38.2 & 1.8 & 10.9 & 1.0 & $14400\left(^{*}\right)$ & 9.3 & $14400(*)$ & 19.4 & 1.0 \\
\hline P.ave. & 37.5 & 6.8 & 11.3 & 3.3 & 13441.1 & 6170.9 & 12961.5 & 6040.7 & 2726.3 \\
\hline Esc32a & 29161.5 & 728.2 & 7095.2 & 638.7 & $14400\left(^{*}\right)$ & $14400(*)$ & $14400(*)$ & $14400(*)$ & - \\
\hline Esc32b & 29176.7 & 1253.3 & 7198.3 & 1071.1 & $14400\left(^{*}\right)$ & $14400\left(^{*}\right)$ & $14400\left(^{*}\right)$ & $14400\left(^{*}\right)$ & - \\
\hline Esc32c & 29186.2 & 1633.2 & 7109.0 & 1363.4 & $14400(*)$ & $14400(*)$ & $14400\left(^{*}\right)$ & $14400\left(^{*}\right)$ & - \\
\hline Esc32d & 29271.5 & 1143.1 & 7131.2 & 1009.5 & $14400\left(^{*}\right)$ & $14400(*)$ & $14400(*)$ & $14400(*)$ & - \\
\hline Esc32e & 29168.1 & 62.8 & 7265.1 & 58.4 & $14400\left(^{*}\right)$ & 5.9 & $14400\left(^{*}\right)$ & $14400\left(^{*}\right)$ & 600.0 \\
\hline Esc32f & 30419.7 & 63.0 & 7135.3 & 58.1 & $14400(*)$ & 5.9 & $14400(*)$ & $14400\left(^{*}\right)$ & 600.0 \\
\hline Esc32g & 29258.3 & 119.3 & 7499.9 & 112.2 & $14400(*)$ & 208.4 & $14400(*)$ & 100.2 & - \\
\hline Esc32h & 29318.7 & 1740.6 & 7510.1 & 1447.0 & $14400(*)$ & $14400\left(^{*}\right)$ & $14400\left(^{*}\right)$ & $14400\left(^{*}\right)$ & - \\
\hline P.ave. & 29370.1 & 842.9 & 7243.0 & 719.8 & $14400\left(^{*}\right)$ & 9027.5 & $14400\left(^{*}\right)$ & 12612.5 & - \\
\hline T.ave. & 7554.9 & 215.3 & 1849.0 & 182.7 & 11883.1 & 4194.8 & 9614.0 & 5063.8 & - \\
\hline
\end{tabular}

${ }^{a}$ For the instances Chr12a - Char25a, the CPU times are obtained with a UNIVAC 1100/60. For the instances Esc16a - Esc16j, the CPU times are obtained with a 16-processor MEIKO Computing Surface with Intel i860 processors. For the instances Esc32e and Esc32f, the CPU times are obtained with NEC Cenju-3.

${ }^{b}$ The CPU time limit, 14400 seconds, was reached. 
flow and a distance, $q_{i j k l}=f_{i k} d_{j l}$, one obtains a sparse matrix if some of the factors are null. In most situations some values $f_{i k}$ are zero, and we exploited this situation here. The results obtained here also apply, by symmetry, to the case where $d_{j l}=0$.

Finally we would like to point out that these results are an example showing that the tighter formulation is not always the most effective to solve a problem. If we consider as a measure the solving time of the IP, or the quality of the integer solution within some time limit, the less tight formulations outperformed the tightest. The technique used here to weaken a formulation to make its LP relaxation much easier to solve may prove fruitful in other applications.

\section{Acknowledgement}

Thanks to the support from the project ADONET-Marie Curie Research Training Networks-Contract Nr MRTN-CT-2003-504438. We wish to thank the Faculty of Sciences of the University of Lisbon for providing the software Cplex 9.0, and thank Peter Hahn for providing the information of the CPU times in the literatures. We also thank the support of the grant URJC-CM-2007-CET-1622 from Rey Juan Carlos University and Comunidad de Madrid (Spain) and the grant MTM200614961-C05-05 from the Spanish government.

\section{References}

[1] W. P. Adams, M. Guignard, P. M. Hahn, and W. L. Hightower. A level-2 reformulation linearization technique bound for the quadratic assignment problem. European Journal of Operational Research, 180(3):983-996, 2007.

[2] W. P. Adams and T. A. Johnson. Improved linear programming-based lower bounds for the quadratic assignment problem. DIMACS Series in Discrete Mathematics and Theoretical Computer Science, American Mathematical Society, 16:43-75, 1994.

[3] R. K. Ahuja, J. B. Orlin, and A. Tivari. A greedy genetic algorithm for the quadratic assignment problem. Computers and Operations Research, 27(10):917934, 2000.

[4] K. Anstreicher, N. Brixius, J.-P. Goux, and J. Linderoth. Solving large quadratic assignment problems on computational grids. Mathematical Programming, 91(3):563-588, 2002.

[5] K. M. Anstreicher. Recent advances in the solution of quadratic assignment problems. Mathematical Programming, 97(1-2):27-42, 2003.

[6] N. W. Brixius and K. M. Anstreicher. Solving quadratic assignment problems using convex quadratic programming relaxations. Optimization Methods and Software, 16(1-4):49-68, 2001. 
[7] A. Brungger, A. Marzetta, J. Clausen, and M. Perregaard. Joining forces in solving large-scale quadratic assignment problem in parallel. 11th International Parallel Processing Symposium (IPPS '97), 1-5 April 1997, Geneva, Switzerland, Proceedings, pages 418-427.

[8] M. J. Brusco and S. Stahl. Using quadratic assignment methods to generate initial permutations for least-squares unidimensional scaling of symmetric proximity matrices. Journal of Classification, 17(2):197-223, 2000.

[9] R. E. Burkard, S. E. Karisch, and F. Rendl. Qaplib-a quadratic assignment problem library. Journal of Global Optimization, 10(4):391-403, 1997.

[10] E. Cela. The Quadratic Assignment Problem: Theory and Algorithms. Kluwer Academic Publishers, London, 1998.

[11] N. Christofides and E. Benavent. An exact algorithm for the quadratic assignment problem. Operations Research, 37(5):760-768, 1989.

[12] J. Clausen and M. Perregaard. Solving large quadratic assignment problems in parallel. Computational Optimization and Applications, 8(2):111-127, 1997.

[13] C. W. Commander. A survey of the quadratic assignment problem, with applications. Morehead Electronic Journal of Applicable Mathematics, (4):1-15, 2005 .

[14] D. T. Connolly. An improved annealing scheme for the qap. European Journal of Operational Research, 46(1):93-100, 1990.

[15] T. G. Crainic, B. Le Cun, and C. Roucairol. Parallel Combinatorial Optimization, chapter 1, Parallel Branch-and-Bound Algorithms, pages 1-28. John Wiley \& Sons, Inc., 2006.

[16] Z. Drezner. Extensive experiments with hybrid genetic algorithms for the solution of the quadratic assignment problem. Computers and Operations Research, 35(3):717-736, 2008.

[17] E. Duman and I. Or. The quadratic assignment problem in the context of the printed circuit board assembly process. Computers and Operations Research, 34(1):163-179, 2007.

[18] G. Erdogan and B. Tansel. A branch-and-cut algorithm for quadratic assignment problems based on linearizations. Computers and Operations Research, 34(4):1085-1106, 2007.

[19] A. M. Frieze and J. Yadegar. On the quadratic assignment problem. Discrete Applied Mathematics, 5(1):89-98, 1983. 
[20] L. M. Gambardella, E. D. Taillard, and M. Dorigo. Ant colonies for the quadratic assignment problem. Journal of the Operational Research Society, 50(2):167176, 1999.

[21] A. M. Geoffrion and G. W. Graves. Scheduling parallel production lines with changeover costs: Practical applications of a quadratic assignment/lp approach. Operations Research, 24(4):595-610, 1976.

[22] P. C. Gilmore. Optimal and suboptimal algorithms for the quadratic assignment problem. SIAM Journal on Applied Mathematics, 10(2):305-313, 1962.

[23] S. W. Hadley, F. Rendl, and H. Wolkowicz. A new lower bound via projection for the quadratic assignment problem. Mathematics of Operations Research, 17(3):727-739, 1992.

[24] P. Hahn, T. Grant, and N. Hall. A branch-and-bound algorithm for the quadratic assignment problem based on the hungarian method. European Journal of Operational Research, 108(3):629-640, 1998.

[25] L. Kaufmann and F. Broeckx. An algorithm for the quadratic assignment problem using benders' decomposition. European Journal of Operational Research, 2(3):204-211, 1978.

[26] T. C. Koopmans and M. J. Beckmann. Assignment problems and the location of economic activities. Econometrica, 25(1):53-76, 1957.

[27] E. L. Lawler. The quadratic assignment problem. Management Science, 9(4):586-599, 1963.

[28] Y. Li, P. M. Pardalos, K. G. Ramakrishnan, and et al. Lower bounds for the quadratic assignment problem. Annals of Operations Research, 50(1):387-441, 1994.

[29] Y. Li, P. M. Pardalos, and M G. C. Resende. A greedy randomized adaptive search procedure for the quadratic assignment problem. DIMACS Series in Discrete Mathematics and Theoretical Computer Science, American Mathematical Society, 16:237-261, 1994.

[30] E. M. Loiola, N. M. M. Abreu, P. O. Boaventura-Netto, and et al. A survey for the quadratic assignment problem. European Journal of Operational Research, 176(2):657-690, 2007.

[31] B. Mans, T. Mautor, and C. Roucairol. A parallel depth first search branch and bound algorithm for the quadratic assignment problem. European Journal of Operational Research, 81(3):617-628, 1995.

[32] A. Misevicius. A modified simulated annealing algorithm for the quadratic assignment problem. Informatica, 14(4):497-514, 2003. 
[33] P. M. Pardalos, F. Rendl, and H. Wolkowicz. The quadratic assignment problem: A survey and recent developments. DIMACS Series in Discrete Mathematics and Theoretical Computer Science, American Mathematical Society, 16:1-42, 1994.

[34] M. A. Pollatschek, N. Gershoni, and Y. T. Radday. Optimization of the typewriter keyboard by simulation. Angewandte Informatik, 17(0):438-439, 1976.

[35] K. G. Ramakrishnan, M. G. C. Resende, and P. M. Pardalos. A branch and bound algorithm for the quadratic assignment problem using a lower bound based on linear programming. In State of the Art in Global Optimization: Computational Methods and Applications, Kluwer Academic Publishers, pages 57-73, 1996.

[36] M. G. C. Resende, K. G. Ramakrishnan, and Z. Drezner. Computing lower bounds for the quadratic assignment problem with an interior point algorithm for linear programming. Operations Research, 43(5):781-791, 1995.

[37] S. Sahni and T. Gonzalez. P-complete approximation problems. Journal of the Association of Computing Machinery, 23(3):555-565, 1976.

[38] Y. Xia. Improved gilmore-lawler bound for quadratic assignment problems. Chinese Journal of Engineering Mathematics, 24(3):401-413, 2007.

[39] Q. Zhao, S. E. Karisch, F. Rendl, and et al. Semidefinite programming relaxations for the quadratic assignment problem. Journal of Combinatorial Optimization, 2(1):71-109, 1998. 